\title{
POLSAR IMAGES CHARACTERIZATION THROUGH BLIND SOURCES SEPARATION TECHNIQUES
}

\author{
Felix Totir, Gabriel Vasile, Lionel Bombrun and Michel Gay \\ Grenoble-Image-sPeach-Signal-Automatics Lab, CNRS / Grenoble-INP \\ 961 rue de la Houille Blanche, BP 46, 38402 Grenoble Cedex, FRANCE
}

\begin{abstract}
Since the backscattered signal in PolSAR images is intrinsically linked with the physical characteristics of the objects in the image, valuable information may be extracted therefrom. The paper focus is to propose a new physical characterization of the scattering target, inspired by the Blind Sources Separation techniques.
\end{abstract}

Index Terms - PolSAR, incoherent decomposition, ICA, PCA.

\section{INTRODUCTION}

Appropriate backscattered signal stochastic models are essential for correctly segmenting high-resolution Polarimetric SAR (PolSAR) images. Modeling using multivariate, centered, circular Gaussian random process is no longer acceptable for the new high quality images, since the reduced dimensions of the resolution cells increase the heterogeneity of the observed scene and obsoletes the Gaussian polarimetric model. Examples of advanced, non-Gaussian polarimetric stochastic models are the SIRV model and various submodels (e.g. the KummerU model [1], which assumes a Fisherdistributed texture).

During classification of image pixels in PolSAR, each pixel receive the label of a class. This is illustrated in Fig. 1 [1] (classes are coded with colors). The segmentation algorithm essentially relies on the underlying stochastic model assumed for the backscattered radar signal (e.g. Gaussian, SIRV/KummerU, etc.). As such, homogeneity of determined classes is based on the statistical distribution of PolSAR image pixels, and the physical characteristics of the objects in the image are not considered at this stage.

In order to complement the stochastically-based pixel classification, physical characterization may be also used. Since the backscattered signal is intrinsically linked with the physical characteristics of the objects in the image, valuable information may be extracted therefrom. The paper focus is to propose a new physical characterization of the scattering

This work is supported through the EFIDIR project and the NATO research grant EAP.RIG.982933 target, inspired by the Blind Sources Separation (BSS) techniques.

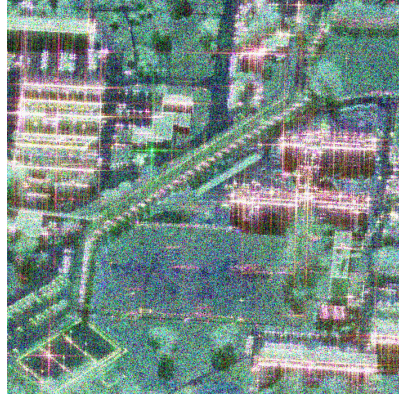

(a)

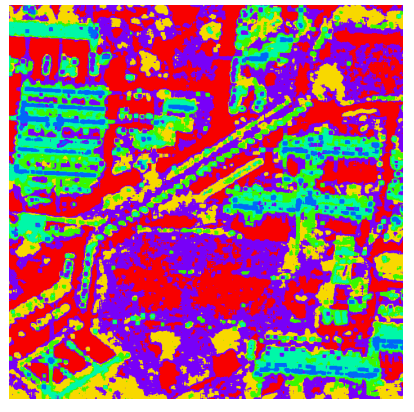

(b)
Fig. 1. Classification results for the X-band RAMSES data over the Toulouse test-site $(700 \times 700$ pixels $):$ (a) Colored composition of the target vector $[k]_{1}-[k]_{3}-[k]_{2}$. (b) Classification using the Wishart criterion.

\section{PHYSICAL CHARACTERIZATION}

Efforts have been made in direction of characterizing the physical structure of the objects observed by a radar from their polarimetric echo. Several such decompositions have been proposed: the Huynen decomposition [2], the H/ $\alpha / \mathrm{A}$ decomposition [3], the Target Scattering Vector Model (TSVM) decomposition [4], etc. The fundamental idea of those decomposition is that each elementary reflector (surface, sphere, dihedral, ...) has a particular backscattering mechanism and that each target may be described as a superposition of such elementary reflectors.

\subsection{H/ $\alpha / A$ and TSVM decompositions}

The scattering matrix $\mathbf{S}$ contains the polarimetric components:

$$
\mathbf{S}=\left[\begin{array}{ll}
S_{H H} & S_{H V} \\
S_{V H} & S_{V V}
\end{array}\right]
$$

For a monostatic radar in a homogeneous environment, the reciprocity theorem [5] ensures that $S_{H V}=S_{V H}$. In this case, the scattering vector is defined as a vectorization of the 
scattering matrix $\mathbf{S}$ represented in the Pauli basis:

$$
\mathbf{k}_{P}=\left[\begin{array}{lll}
S_{H H}+S_{V V} & S_{H H}-S_{V V} & 2 S_{H V}
\end{array}\right]^{T} .
$$

The coherency matrix $\mathbf{T}$ is defined as:

$$
\mathbf{T}=E\left[\mathbf{k}_{P} \cdot \mathbf{k}_{P}^{\dagger}\right]
$$

Several incoherent target decompositions (ICTD) theorems have been proposed in the literature to compute polarimetric parameters from the coherency matrix $\mathbf{T}$. The $\mathrm{H} / \alpha / \mathrm{A}$ and TSVM-ICTD decompositions extract a first set of parameters computed from the eigenvalues of $\mathbf{T}$, and a second set of parameters which is computed from the eigenvectors of $\mathbf{T}$, using either the $\alpha / \beta$ model (for the $\mathrm{H} / \alpha / \mathrm{A}$ decomposition) [3] or the TSVM model [4].

Since $\mathbf{T}$ is hermitian and positive semi-definite, it is possible to write:

$$
\mathbf{T}=\mathbf{V} \boldsymbol{\Sigma} \mathbf{V}^{\dagger}=\sum_{i=1}^{3} \lambda_{i} \mathbf{k}_{i} \mathbf{k}_{i}^{\dagger},
$$

where $\boldsymbol{\Sigma}$ is the diagonal matrix of the eigenvalues of $\mathbf{T}$ (arranged in decreasing order), while $\mathbf{V}$ is the complex matrix whose columns are the corresponding eigenvectors of $\mathbf{T}$.

\subsection{Parametrization of eigenvalues}

Three roll-invariant parameters can be extracted from the eigenvalues of $\mathbf{T}$, namely the span: $\operatorname{span}=\sum_{i=1}^{3} \lambda_{i}$; the entropy: $H=\sum_{i=1}^{3}-p_{i} \log _{3} p_{i}$ where the pseudo-probabilities $p_{i}$ are given by $p_{i}=\frac{\lambda_{i}}{\sum_{j=1}^{3} \lambda_{j}}$ and the anisotropy: $A=$ $\frac{\lambda_{2}-\lambda_{3}}{\lambda_{2}+\lambda_{3}}=\frac{p_{2}-p_{3}}{p_{2}+p_{3}}$.

\subsection{Parametrization of eigenvectors}

The parameters computed from the eigenvectors vary according to the retained model.

In the $\alpha / \beta$ model [3], each eigenvector $\mathbf{k}_{\mathbf{i}}$ of the coherency matrix $\mathbf{T}$ is parametrized using five angles $\left(\alpha_{i}, \beta_{i}, \gamma_{i}, \delta_{i}, \theta_{i}\right)$ :

$$
\mathbf{k}_{\mathbf{i}}=e^{j \theta_{i}}\left[\begin{array}{c}
\cos \alpha_{i} \\
\sin \alpha_{i} \cos \beta_{i} e^{j \delta_{i}} \\
\sin \alpha_{i} \sin \beta_{i} e^{j \gamma_{i}}
\end{array}\right] .
$$

Global characterization of the target is achieved with weighted averaged parameters (e.g. $\alpha=p_{1} \alpha_{1}+p_{2} \alpha_{2}+$ $p_{3} \alpha_{3}$ ). For the $\alpha / \beta$ model, only $\alpha_{i}$ angles are roll-invariant. The (incoherent) $\mathrm{H} / \alpha / \mathrm{A}$ decomposition reunites the $H, \alpha$ and $A$ parameters.

The (incoherent) TSVM decomposition, proposed by Touzi in 2007, consists in the following parametrization of coherence matrix $\mathbf{T}$ eigenvectors $\mathbf{k}_{\mathbf{i}}$ (see [4] for details). It leads:

$$
\begin{aligned}
\mathbf{k}_{\mathbf{i}} & =e^{j \Phi_{s_{i}}}\left[\begin{array}{ccc}
1 & 0 & 0 \\
0 & \cos \left(2 \psi_{i}\right) & -\sin \left(2 \psi_{i}\right) \\
0 & \sin \left(2 \psi_{i}\right) & \cos \left(2 \psi_{i}\right)
\end{array}\right] \\
& \times\left[\begin{array}{c}
\cos \alpha_{s_{i}} \cos \left(2 \tau_{m_{i}}\right) \\
\sin \alpha_{s_{i}} e^{j \Phi_{\alpha_{s_{i}}}} \\
-j \cos \alpha_{s_{i}} \sin \left(2 \tau_{m_{i}}\right)
\end{array}\right] .
\end{aligned}
$$

$\psi_{i}$ is the orientation of the maximum polarization with respect to the horizontal polarization. $\tau_{m}$ is the target helicity, it characterizes the symmetry of the target. $\alpha_{s}$ and $\Phi_{\alpha_{s}}$ are the symmetric scattering type magnitude and phase. They are derived from the con-eigenvalues $\mu_{1}$ and $\mu_{2}$ of the scattering matrix $\mathbf{S}$ by:

$$
\tan \left(\alpha_{s}\right) e^{j \Phi_{\alpha_{s}}}=\frac{\mu_{1}-\mu_{2}}{\mu_{1}+\mu_{2}} .
$$

The TSVM decomposition gives four roll-invariant (independent of $\psi_{i}$ ) parameters for each eigenvector, namely $\alpha_{s_{i}}$, $\Phi_{\alpha_{s_{i}}}, \tau_{m_{i}}$ and $\lambda_{i}$.

\section{BLIND SOURCES SEPARATION TECHNIQUES}

An alternative to the physical decompositions based on the covariance matrix is to use the techniques dedicated for blind source separation (BSS). In its simplest form, the latter assumes that several sensors record different linear combinations of an equal number of sources. Making use of relatively weak hypothesis on the original sources (e.g. the statistical independence of the random sources), the BSS techniques attempt to retrieve the coefficients of the linear combinations (e.g. the mixing matrices) and, correspondingly, the signals transmitted by the sources.

\subsection{ICA- and PCA-decomposition}

Under the hypothesis that PolSAR image pixels are realizations of a multivariate (three-dimensional) stochastic process (for example, the KummerU model [1]), whose components are linear combinations of one-dimensional sources, the scattering vectors may be written as:

$$
\mathbf{k}_{P}=\mathbf{A}\left[\begin{array}{c}
s_{1} \\
s_{2} \\
s_{3}
\end{array}\right]=\mathbf{B}\left[\begin{array}{l}
v_{1} \\
v_{2} \\
v_{3}
\end{array}\right]
$$

In the equation above, sources $s_{1}, s_{2}$ and $s_{3}$ (forming vector s) are assumed to be statistically independent, while sources $v_{1}, v_{2}$ and $v_{3}$ (forming vector $\mathbf{v}$ ) are assumed to be statistically uncorrelated. Obviously, the latter hypothesis is weaker, since components of $\mathbf{s}$ are also uncorrelated. More, components of $\mathbf{s}$ and $\mathbf{v}$ are assumed to have unit power (their correlation matrices are the identity matrix). $\mathbf{A}$ and $\mathbf{B}$ are the corresponding $3 \times 3$ mixing matrices. There are those matrices that 
bear information about the relative energies of components in vectors $\mathbf{s}$ and $\mathbf{v}$.

One should note that the decompositions above are not always pertinent since not always the components of $\mathbf{k}_{P}$ are obtained through linear mixing of some statistically independent (or statistically uncorrelated) random sources. In the particular case of PolSAR images, those sources should represent elementary scattering mechanisms (dihedral angle, surface, etc.). The presence of those scattering mechanism in a considered pixel (cell resolution) is debatable.

Based on the statistical assumptions regarding components of $\mathbf{s}$ and $\mathbf{v}$, the decomposition As is called ICAdecomposition and, respectively, the decomposition $\mathbf{B v}$ is called PCA-decomposition. The relations between T, A and $B$ are:

$$
\begin{aligned}
\mathbf{T} & =\mathbf{A A}^{\dagger}=\mathbf{B B}^{\dagger} \\
\mathbf{A} & =\mathbf{B P} \\
\mathbf{P P}^{\dagger} & =\mathbf{I}_{\mathbf{3}}
\end{aligned}
$$

Basically, both A and B are factorizations ("square roots") of matrix $\mathbf{T}$. The unitary matrix $\mathbf{P}$ is assimilable to a rotation. Mixing matrices $\mathbf{A}$ and $\mathbf{B}$ are not known, but they may be retrieved under two paradigms.

First, since the only constraint placed on $\mathbf{B}$ is described in second-order statistics (that correlation matrix of $\mathbf{v}$ is identity matrix), it follows that $\mathbf{B}$ is retrievable through an analysis of the second-order statistics (i.e. the coherency matrix $\mathbf{T}$ ). Indeed, it follows that choosing the columns of $\mathbf{B}$ to be the very eigenvectors of $\mathbf{T}$ (more precisely, their un-normalized versions, i.e. multiplied with square roots of corresponding eigenvalues) is such a sufficient constraint.

However, the choice made above is not unique, since there are many other choices that ensure uncorrelation between components of $\mathbf{v}$ : multiplication of $\mathbf{B}$ with an arbitrary but unitary matrix $\mathbf{P}$ still ensures lack of correlation between components of $\mathbf{v}$.

On the other hand, choosing the eigenvectors of $\mathbf{T}$ for the columns of $\mathbf{B}$ ensures the fact that maximum energies are achieved for components of $\mathbf{v}$. From the particular structure of $\mathbf{T}$ it also follows that the eigenvectors are orthogonal. These properties are lost through multiplication with $\mathbf{P}$.

Second, matrix A cannot be retrieved under the same paradigm, since statistical independence cannot be tested in the restricted framework of second-order statistics, unless multivariate Gaussian is assumed. Hence, while intrinsically linked to $\mathbf{T}, \mathbf{A}$ cannot be retrieved through an analysis of $\mathbf{T}$ only. Equivalently, one could say that the "floating" matrix $\mathbf{P}$ cannot be determined from $\mathbf{T}$ (there is no link between $\mathbf{P}$ and T).

However, under some fairly weak assumptions (nonsymmetric probability densities of $\mathbf{s}$ ), matrix $\mathbf{A}$ can be estimated under the BSS paradigm, through ICA. While this technique is rather classical today, extra-care is required when dealing with complex random vectors. The method used in this paper is FastICA [6]. This is a rapidly converging (it uses a fixed-point iteration scheme) algorithm, maximizing the non-Gaussianity as a measure of statistical independence.

\subsection{Reworking the H/A, $\alpha / \beta$ and TSVM decompositions}

Here we present a reformulation of the incoherent decompositions given in section 2.1. The goal is to drop references to the matrix $\mathbf{T}$ and to give instead formulations based on equation 8. which will create an unifying framework. It is assumed that $\mathbf{B}$ is chosen so that its columns are the weighted eigenvectors of $\mathbf{T}$. In this case, the decompositions in section 2.1 remain basically unchanged. However, supplanting the PCAdecomposition with the ICA-decomposition is also envisaged here. This will lead to new, alternative decompositions.

\subsubsection{Reworking the H/A model}

Both $\mathrm{H}$ and $\mathrm{A}$ are computed from the eigenvalues of $\mathbf{T}$. These eigenvalues are none others than the energies (squared norms) of columns of $\mathbf{B}$ or, equivalently, the diagonal elements of matrix $\mathbf{B}^{\dagger} \mathbf{B}$. Actually, $\mathrm{H}$ and $\mathrm{A}$ are identical with their PCAbased counterparts.

On the other hand, it is envisaged to use the energies (squared norms) of columns of $\mathbf{A}$ instead of eigenvalues of $\mathbf{T}$ when computing $\mathrm{H}$ and $\mathrm{A}$. This leads to ICA-based versions of $\mathrm{H}$ and $\mathrm{A}$ and will be no longer identical to their PCA-based counterparts. More, since columns of $\mathbf{A}$ are no longer orthogonal in the ICA-based versions, a somewhat distorted information is expected (information contained in non-diagonal elements of matrix $\mathbf{A}^{\dagger} \mathbf{A}$ is lost).

\subsubsection{Reworking the $\alpha / \beta$ and TSVM models}

The $\alpha / \beta$ and TSVM models rely on parameterizing the eigenvectors of $\mathbf{T}$. These are identical to the columns of $\mathbf{B}$, normalized (to have unit energy). Hence, the PCA-based versions, which use the normalized columns of $\mathbf{B}$ are identical to the classical $\alpha / \beta$ and TSVM models. On the other hand, the ICA-based versions (parameterizing the normed columns of $\mathbf{A})$ will give different results, since the columns of $\mathbf{A}$ are not the same as columns of $\mathbf{B}$.

\section{RESULTS}

\subsection{On a synthetic data-set}

In this section, a synthetic data-set is analyzed. Let $\mathrm{A}$ be the mixing matrix of dimension $3 \times 3$. This matrix is composed by three target vector, namely $k_{1}, k_{2}$ and $k_{3}$, which represents three different scattering mechanisms. (10) shows the roll-invariant mixing matrix, denoted $\mathrm{A}^{\text {roll-inv }}$, i.e. the mixing matrix which contains the three roll-invariant target vector. Three sources $\left(s_{1}, s_{2}\right.$ and $\left.s_{3}\right)$ issued respectively from three 
different distributions are simulated. Next, the observed signal $\mathbf{k}_{P}=$ As is formed, where $\mathbf{s}=\left[s_{1}, s_{2}, s_{3}\right]^{T}$.

$$
\begin{aligned}
\mathrm{A}^{\text {roll-inv }} & =\left[k_{1}^{\text {roll-inv }}, k_{2}^{\text {roll-inv }}, k_{3}^{\text {roll-inv }}\right] \\
& =\left[\begin{array}{ccc}
0.901 & 0.433 & 0.294 \\
0.217+0.376 i & 0.470-0.171 i & 0.294+0.096 i \\
0 & 0.750 i & -0.905 i
\end{array}\right]
\end{aligned}
$$

ICA and PCA decompositions are next applied on this synthetic data-set. (11) and (12) shows the roll-invariant estimated mixing matrix for respectively the ICA $\left(\hat{\mathrm{A}}_{\mathrm{ICA}}^{\text {roll-inv }}\right)$ and the PCA decomposition ( $\hat{\mathrm{A}}_{\mathrm{PCA}}^{\text {roll-inv }}$ ). As the three sources are statistically independent, the ICA decomposition is able to retrieve perfectly the three scattering mechanism. Nevertheless, as the three target scattering vector are not orthogonal, the PCA decomposition fails as observed in (12). The ICA decomposition gives another interpretation of the PolSAR images.

$$
\hat{\mathrm{A}}_{\mathrm{ICA}}^{\text {roll-inv }}=
$$

$$
\begin{gathered}
=\left[\begin{array}{ccc}
0.170 & 0.386 & 0.901 \\
0.310-0.126 i & 0.489-0.118 i & 0.216+0.377 i \\
-0.927 i & 0.773 i & -0.001 i
\end{array}\right] . \\
\hat{\mathrm{A}}_{\mathrm{PCA}}^{\text {roll-inv }}= \\
=\left[\begin{array}{ccc}
0.437 & 0.250 & 0.864 \\
-0.484+0.665 i & -0.688+0.631 i & 0.194-0.464 i \\
-0.365 i & 0.259 i & 0.007 i
\end{array}\right] .
\end{gathered}
$$

\subsection{On a real data-set}

In this part, the X-band RAMSES data-set over the Toulouse test-site shown in Fig. 1(a) is analyzed. The ICA decomposition is applied on a $7 \times 7$ sliding square window. Fig. 2 shows the four roll-invariant parameters $\left(\alpha, \alpha_{s}, \Phi_{\alpha_{s}}\right.$ and $\left.\tau_{m}\right)$ extracted from the dominant component.

\section{CONCLUSIONS AND PERSPECTIVES}

The paper presented a re-formulation (and an extension) of the incoherent PolSAR H/A, $\alpha / \beta$ and TSVM decompositions in terms of Principal Component and Independent Component analysis. For the former, the decompositions reduce to their classical formulation but, for the latter, they give different results. Future work should be directed at a more thorough analysis of the information obtained from ICA-based decompositions.

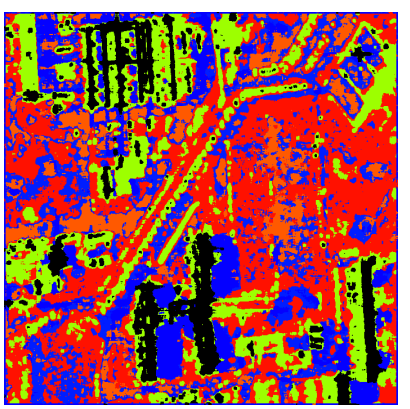

(a)

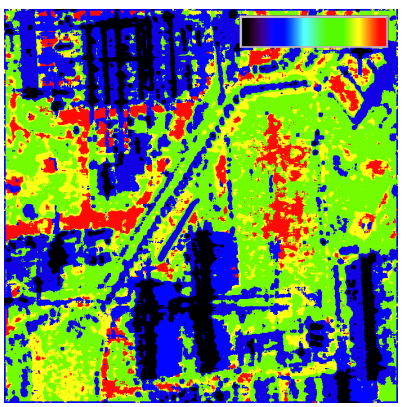

(c)

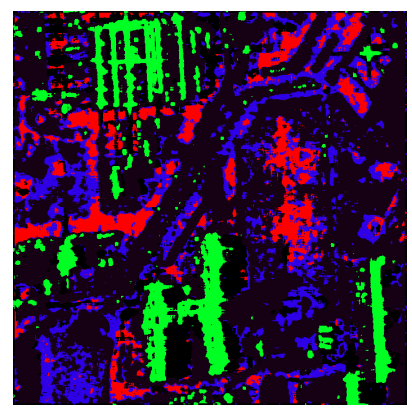

(b)

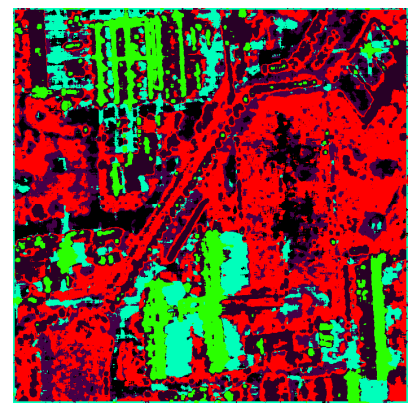

(d)
Fig. 2. ICA results for the X-band RAMSES data over the Toulouse test-site $(700 \times 700$ pixels). Roll-invariant parameters issued from the dominant component: (a) $\alpha$. (b) $\alpha_{s}$, (c) $\Phi_{\alpha_{s}}$, (d) $\tau_{m}$.

\section{REFERENCES}

[1] L. Bombrun, G. Vasile, and M. Gay, "Segmentation of Polarimetric SAR images based on the SIRV model and the KummerU PDF," in 22e colloque GRETSI sur le Traitement du Signal et des Images, GRETSI 2009, Dijon, France, 2009.

[2] J.R. Huynen, Phenomenological Theory of Radar Targets, Academic Press, 1978.

[3] S.R. Cloude and E. Pottier, "An Entropy Based Classification Scheme for Land Applications of Polarimetric SAR," IEEE Transactions on Geoscience and Remote Sensing, vol. 35, no. 1, pp. 68-78, 1997.

[4] R. Touzi, "Target Scattering Decomposition in Terms of Roll-Invariant Target Parameters," IEEE Transactions on Geoscience and Remote Sensing, vol. 45, no. 1, pp. 7384, January 2007.

[5] F.T. Ulaby and C. Elachi, Radar Polarimetry for Geoscience Applications, Artech House, 1990.

[6] A. Hyvarinen, "Fast and robust fixed-point algorithms for independent component analysis," IEEE Transactions on Neural Networks, vol. 3, no. 10, pp. 626-634, 1999. 